\title{
DETERMINACIÓN DE MEDIOS DE CULTIVO Y PH PARA LA MASIFICACIÓN in vitro DE CEPAS DE Suillus luteus Aubl. ASOCIADAS A Pinus radiata D. Don Y Scleroderma citrinum Pers. ASOCIADAS A Eucalyptus globulus Labill. DE LA REGIÓN DEL BIOBÍO, CHILE ${ }^{1}$
}

\begin{abstract}
Marta González ${ }^{2}$ Iván Quiroz ${ }^{3}$, Ruy Travieso ${ }^{4}$, Patricio Chung $^{5}$ e Edison García ${ }^{6}$
RESUMEN - El uso de agentes biológicos en la producción en vivero ha aflorado como un elemento que permite no sólo mejorar la calidad morfológica y fisiológica de las plantas, sino que también posibilitar un mayor éxito en el ámbito silvicultural de una plantación. Estos agentes biológicos requieren de un ambiente óptimo para crecer, asociarse y reproducirse. El objetivo de este estudio fue determinar la dependencia del $\mathrm{pH}$ y el medio de cultivo en el crecimiento in vitro de distintas cepas de Suillus luteus y Scleroderma citrinum asociadas a Pinus radiata y Eucalyptus globulus, respectivamente. El estudio se realizó en condiciones controladas de temperatura y humedad, disponiendo los inóculos en placas Petri con diferentes medios cultivo, evaluando parámetros de crecimiento y biomasa a los 38 días para S. luteus y 105 días para S. citrinum. Los resultados indican que tanto el medio de cultivo, el pH del medio, así como las cepas de cada especie estudiada, son determinantes en las respuestas de crecimiento de los hongos ectomicorrícicos in vitro evaluados. Las cepas de S. luteus se desarrollaron adecuadamente en un medio de cultivo con abundancia de nutrientes (BAF, MMN) como en baja presencia de ellos (EMA), y con pH más bien ácido $(4,8$ y 5,8). Por otro lado, las cepas de $S$. citrinum presentaron, para los mismos ambientes, un desarrollo inferior y lento, no obstante, la cepa Sc8 se reprodujo de forma óptima y rápida bajo un medio de cultivo BAF y con un $\mathrm{pH}$ moderadamente ácido de 5,8.
\end{abstract}

Palabras-clave: Hongos micorrícicos; Efecto del pH; Medios de cultivo.

\section{DETERMINAÇÃO DE MEIOS DE CULTIVO E PH PARA A MASSIFICAÇÃO in vitro DE CEPAS DE Suillus luteus Aubl. SÓCIA A Pinus radiata D. Don e Scleroderma citrinum Pers. ASSOCIADA A Eucalyptus globulus Labill. DA REGIÃO DO BIOBÍO, CHILE}

\begin{abstract}
RESUMO - O uso de agentes biológicos na produção em vivero tem surgido como elemento que permite não só melhorar a qualidade morfológica e fisiológica das plantas, como também posibilitar maior sucesso no ámbito silvicultural de uma plantação. Esses agentes biológicos requerem ambiente ótimo para crescer, se associar e reproduzir. O objetivo deste estudo foi determinar a dependência do pH e a média de cultivo no crescimento in vitro de diferentes cepas de Suillus luteus e Scleroderma citrinum associadas a Pinus radiata e Eucalyptus globulus, respectivamente. O estudo foi realizado sob condições de temperatura e umidade controladas, com o inóculo em placas de Petri com diferentes meios de cultura e avaliação de parâmetros de crescimento de biomassa por 38 dias para S. luteus e 105 dias para S. citrinum. Os resultados indicaram que tanto o meio de cultivo quanto o pH do meio, bem como as cepas da cada espécie estudada, são determinantes nas respostas de crescimento dos fungos ectomicorrízicos in vitro avaliados. As cepas de Suillus luteus desenvolveram-se adequadamente num meio de cultivo com abundância de nutrientes (BAF e MMN), como baixa presença
\end{abstract}

\footnotetext{
${ }^{1}$ Recebido em 07.08.2013 aceito para publicação em 08.10.2014.

${ }^{2}$ Instituto Forestal, Concepción, Región del Biobío, Chile. E-mail:<mgonzale@infor.cl>,<iquiroz@infor.cl>, <ruytravieso@gmail.com>, <pchung@infor.cl>e <egarcia@infor.cl>.
} 
deles (EMA) e com pH bem mais ácido (4,8 e 5,8). Entretanto, as cepas de Scleroderma citrinum apresentaram, nos mesmos ambientes, desenvolvimento inferior e lento, não obstante a cepa Sc8 ter-se reproduzido, de forma ótima e rápida, baixo meio de cultivo BAF e pH moderadamente ácido 5,8.

Palavras-chave: Fungos micorrízicos; Efeito do pH; Meios de cultura.

\section{INTRODUCCIÓN}

En los últimos años se han desarrollado mejoras tecnológicas encaminadas a conseguir una planta de calidad para incrementar la productividad de una plantación en términos de sobrevivencia y crecimiento. Ejemplo de ello es lo mencionado por Schmid(2006) con Pinus cembra L. inoculado, en plantaciones establecidas a gran altitud demostrándose un aumento de $20-80 \%$ en la supervivencia cuando, incrementándose a 50-90\% cuando la inoculación se combina con técnicas de silvicultura intensiva.Uno de los puntos de mayor relevancia es la condición del sistema radicular de la planta, la que debe ser tal que le permita absorber la humedad y nutrientes del suelo de manera más eficiente. Este hecho lo confirman Smith et al.(2004), quienes mencionan que hasta el 100\% de la incorporación del fosfato puede ser responsabilidad de las asociaciones micorrícicas. Es por ello que el uso de agentes biológicos, en particular, la micorrización controlada, es una herramienta que ha aflorado como un elemento que permite no sólo mejorar la calidad morfológica y fisiológica de las plantas en vivero, sino que también una mejor respuesta en plantación, especialmente en suelos degradados (DUÑABEITIA et al., 2004).

Se estima que aproximadamente el 95\% de las plantas en sus hábitats naturales forman una relación mutualista con micorrizas y que los filamentos de hongos pueden llegar a explorar entre 100 a 1.000 veces más tierra que las propias raíces de las plantas (HONRUBIA et al., 1992; BRUNDRETT et al., 1996). Esta relación mutualista se logra mediante la colonización de las micorrizas en las raíces de las plantas, la que puede ser de forma extracelular o intracelular. La relación mutualista provee al hongo prácticamente, y de manera constante y directa, entre el 10 y el $30 \%$ del total de carbohidratos (monómericos y diméricos), producidos en la fotosíntesis $\mathrm{y}$, de vitaminas metabolizadas en otros procesos internos de la planta. Por su parte la planta puede utilizar la amplia red de micelios del hongo en orden de absorber agua y/o nutrientes desde el suelo, mejorando su capacidad de captación de estos elementos. Las micorrizas son especialmente beneficiosas para plantas en terrenos pobres en nutrientes, y se ha demostrado que entrega también resistencia a ciertos patógenos y mayor tolerancia a la sequía (GIANINAZZI-PERSON; AZCON-AGUILAR, 1991; HONRUBIA et al., 1992).

La producción de micorrizas puede ser inducida con técnicas que emplean micelio de hongo cultivado en medios nutritivos, micelio encapsulado en polímeros de alginato, o mediante suspensiones esporales (HONRUBIA et al., 1992; PERA; PARLADÉ, 2005).

Sin embargo, en algunas especies, la producción de inóculo miceliar en ambiente controlado presenta problemas de escaso crecimiento o heterogeneidad de desarrollo entre los subcultivos, ya sea por el tipo de medio de cultivo utilizado, $\mathrm{pH}$ del mismo medio, y entre otro factor, la especie fúngica estudiada así como las diversas cepas de ella (SANTIAGO-MARTÍNEZ et al., 2003; FERNÁNDEZ-MIRANDA et al., 2008; SANTELICES et al., 2012).

El objetivo de este estudio fue determinar la dependencia del $\mathrm{pH}$ y el medio de cultivo en el crecimiento in vitro de distintas cepas de Suillus luteus (L. ex Fr.) Gray y Scleroderma citrinum Pers., asociadas a Pinus radiata D. Don y Eucalyptus globulus Labill., respectivamente.

\section{MATERIALES Y METODOS}

\subsection{Material fúngico}

Se emplearon inóculos del banco de cepas del Instituto Forestal de Chile (Tabla 1), que corresponden a cepas de hongos colectadas desde plantaciones de Pinus radiata y Eucalyptus globulus, ubicadas en la región del Biobío, Chile.

\subsection{Medios de cultivo}

Los medios de cultivo utilizados fueron Extracto de Malta Agar (EMA), Sabouraud Agar (SAB); Medio Melin-Norkrans (MMN)(MARX, 1969),y Biotina Aneurina Ácido Fólico (BAF)(MOSER, 1960), según la metodología aplicada por Santiago-Martínez et al. (2003). El ajuste del pH de los medios de cultivo fue 
Tabla 1 - Caracterización de cepas utilizadas en este estudio. Tabela 1 - Caracterização de estirpes utilizadas neste estudo.

\begin{tabular}{|c|c|c|c|c|c|c|c|c|}
\hline \multirow{2}{*}{ Código } & \multirow{2}{*}{ Especie } & \multirow{2}{*}{ Provincia } & \multirow{2}{*}{ Comuna } & \multicolumn{2}{|c|}{ Coordenadas* } & \multirow{2}{*}{$\begin{array}{c}\text { Suelo } \\
\text { asociada }\end{array}$} & \multirow{2}{*}{ Especie } & \multirow{2}{*}{$\begin{array}{c}\text { Edad del } \\
\text { rodal (años) }\end{array}$} \\
\hline & & & & UTM W & UTM S & & & \\
\hline IF 81008 & Suillus luteus & Arauco & Curanilahue & 643407 & 5861048 & $\begin{array}{c}\text { Franco } \\
\text { Arcilloso }\end{array}$ & $\begin{array}{l}\text { Pinus } \\
\text { radiata }\end{array}$ & 12 \\
\hline IF83009 & Suillus luteus & Ñuble & Ninhue & 698692 & 5860069 & $\begin{array}{c}\text { Franco } \\
\text { Arcilloso }\end{array}$ & $\begin{array}{c}\text { Pinus } \\
\text { radiata }\end{array}$ & 13 \\
\hline IF 88003 & Suillus luteus & Biobío & Antuco & 760724 & 5854430 & Arenoso & Pinus radiata & 6 \\
\hline Sc8 & $\begin{array}{c}\text { Scleroderma } \\
\text { citrinum }\end{array}$ & Arauco & Cañete & 641655 & 5812477 & $\begin{array}{l}\text { Franco } \\
\text { Arcilloso }\end{array}$ & $\begin{array}{c}\text { Eucalyptus } \\
\text { globulus }\end{array}$ & Monte bajo \\
\hline IF84005 & $\begin{array}{l}\text { Scleroderma } \\
\text { citrinum }\end{array}$ & Concepción & $\begin{array}{l}\text { San Pedro } \\
\text { de la Paz }\end{array}$ & 666583 & 5920347 & Arenoso & $\begin{array}{l}\text { Eucalyptus } \\
\text { globulus }\end{array}$ & 4 \\
\hline
\end{tabular}

*: Datum: WGS84; Huso: 18.

realizado con un electrodo de $\mathrm{pH}$ tradicional y se utilizó $\mathrm{HCl}$ para acidificar el medio y $\mathrm{KOH}$ para basificarlo.

\subsection{Establecimiento del ensayo}

E1 ensayo se estableció en laboratorio, con condiciones ambientales de $23^{\circ} \mathrm{C}$ de temperatura y $55 \%$ de humedad de acuerdo a lo indicado por SantiagoMartínez et al. (2003). Cada medio de cultivo testeado se dispuso en placa Petri en cantidad de $15 \mathrm{ml}$, sobre el cual se incorporaron los inóculos de aproximadamente $1 \mathrm{~cm}^{2}$, permaneciendo en cámara de crecimiento por 38 días en el caso de las cepas de Suillus luteus y 105 días en el caso de las cepas de Scleroderma citrinum, debido a las heterogéneas velocidades de crecimiento entre una especie y otra.

\subsection{Cuantificación de crecimiento}

Se cuantificó el crecimiento radial, biomasa (peso seco) y velocidad de crecimiento. La medición del crecimiento radial consistió en trazar en cada placa Petri 4 direcciones a partir del centro donde fue posicionado el inoculo, registrando en cada ocasión el incremento en milímetros del inóculo en su plano horizontal, sobre cada uno de estos ejes.

Los valores de biomasa se obtuvieron al término del período de medición, para ello se extrajo el micelio desde el agar derretido con calentador, y se dispuso el tejido miceliar en un horno a $90^{\circ} \mathrm{C}$ por 72 horas para obtener su peso seco.

Para la estimación de la velocidad de crecimiento, se emplearon los datos obtenidos del crecimiento radial, los cuales fueron analizados y ajustados por medio de una regresión lineal.

\subsection{Análisis estadístico}

El estudio se estableció bajo un diseño completo al azar, considerando 5 repeticiones para cada prueba. Las evaluaciones se realizaron mediante análisis de varianza (ANDEVA) utilizando el software estadístico InfoStat versión 2011/P. La homogeneidad de varianza fue evaluada mediante la prueba de Levene $(\mathrm{P} \leq 0,05)$. El supuesto de normalidad de los residuos se evaluó mediante la prueba de Shapiro-Wilks ( $\mathrm{P} \leq 0,05)$. Para detectar diferencias significativas entre los tratamientos, se realizó la prueba de comparación múltiple Scott y Knott (1974), con un $95 \%$ de confianza. En cada caso, los factores considerados para el análisis fueron Medio de cultivo, $\mathrm{pH}$ y Cepa y la interacción bifactorial de ellos.

Cabe señalar que solo los datos del ensayo de Suillus luteus, cumplieron los supuestos para ser analizados mediante un análisis de varianza tradicional. Por su parte, en el ensayo de Scleroderma citrinum, las interacciones de las variables no cumplieron los supuestos por lo que en este caso, solo se presentan tendencias y medias.

\section{RESULTADOS}

\subsection{Crecimiento Radial}

\subsubsection{Suillus luteus}

La evaluación de las variables en forma aislada indica que, al término del período de estudio, los mayores valores de crecimiento radial se observaron en la cepa IF88003 ( $\mathrm{p} \leq 0,05)$ (Tabla 2). Esta respuesta ocurre con niveles de $\mathrm{pH}$ más ácidos, no sólo con medios de cultivo ricos sino que también pobres en elementos (BAF; EMA, respectivamente) $(\mathrm{p} \leq 0,05)$ (Tabla 2$)$.

Revista Árvore, Viçosa-MG, v.39, n.1, p.105-113, 2015 
Tabla 2 - Valores medios de crecimiento radial ( $\mathrm{mm}$ ), biomasa ( $\mathrm{g}$ ) y velocidad de crecimiento ( $\mathrm{mm} /$ día) para ensayo de Suillus luteus y Scleroderma citrinum por cada variable evaluada.

Tabela 2 - Valores médios de crescimento radial ( $\mathrm{mm}$ ), biomassa $(\mathrm{g})$ e taxa de crescimento ( $\mathrm{mm} /$ dia) nos testes de Suillus luteus e Scleroderma citrinum de cada variável.

\begin{tabular}{|c|c|c|c|c|c|c|}
\hline Especie & Variable & & $\mathrm{n}$ & $\begin{array}{l}\text { Crecimiento } \\
\text { radial } \pm \mathrm{DE}\end{array}$ & $\begin{array}{l}\text { Biomasa } \\
\pm \mathrm{DE}\end{array}$ & $\begin{array}{c}\text { Velocidad } \\
\text { crecimiento } \pm \mathrm{DE}\end{array}$ \\
\hline \multirow{10}{*}{ Suillus luteus } & \multirow{3}{*}{ Cepa } & IF 81008 & 60 & $21,11 \pm 5,67 b$ & $0,10 \pm 0,05 \mathrm{a}$ & $0,56 \pm 0,15 b$ \\
\hline & & IF 83009 & 60 & $22,09 \pm 4,30 \mathrm{~b}$ & $0,10 \pm 0,05 \mathrm{a}$ & $0,58 \pm 0,11 b$ \\
\hline & & IF 88003 & 60 & $26,17 \pm 6,46 a$ & $0,11 \pm 0,04 \mathrm{a}$ & $0,69 \pm 0,17 \mathrm{a}$ \\
\hline & \multirow{4}{*}{$\mathbf{p H}$} & 4,8 & 60 & $24,60 \pm 4,90 \mathrm{a}$ & $0,11 \pm 0,04 \mathrm{a}$ & $0,65 \pm 0,13 a$ \\
\hline & & 5,8 & 60 & $24,14 \pm 4,96 \mathrm{a}$ & $0,11 \pm 0,04 \mathrm{a}$ & $0,64 \pm 0,13 \mathrm{a}$ \\
\hline & & 6,8 & 60 & $20,65 \pm 6,96 b$ & $0,09 \pm 0,05 \mathrm{a}$ & $0,54 \pm 0,18 b$ \\
\hline & & $\mathrm{BAF}$ & 45 & $23,51 \pm 3,00 \mathrm{a}$ & $0,14 \pm 0,04 \mathrm{a}$ & $0,62 \pm 0,08 \mathrm{a}$ \\
\hline & \multirow{3}{*}{$\begin{array}{l}\text { Medio de } \\
\text { cultivo }\end{array}$} & EMA & 45 & $25,64 \pm 6,21 \mathrm{a}$ & $0,11 \pm 0,04 \mathrm{~b}$ & $0,67 \pm 0,16 \mathrm{a}$ \\
\hline & & $\mathrm{MMN}$ & 45 & $22,55 \pm 6,52 b$ & $0,07 \pm 0,03 \mathrm{~d}$ & $0,59 \pm 0,17 \mathrm{~b}$ \\
\hline & & $\mathrm{SAB}$ & 45 & $20,69 \pm 6,37 \mathrm{~b}$ & $0,09 \pm 0,04 \mathrm{c}$ & $0,54 \pm 0,17 b$ \\
\hline \multirow{9}{*}{ Scleroderma citrinum } & \multirow[t]{3}{*}{ Сepa } & IF 84005 & 33 & $16,20 \pm 4,49 \mathrm{~b}$ & $0,22 \pm 0,10 \mathrm{a}$ & $0,15 \pm 0,04 b$ \\
\hline & & Sc8 & 8 & $29,16 \pm 14,74 \mathrm{a}$ & $0,20 \pm 0,08 \mathrm{a}$ & $0,28 \pm 0,14 \mathrm{a}$ \\
\hline & & 4,8 & 4 & $15,84 \pm 5,51 \mathrm{a}$ & $0,17 \pm 0,09 \mathrm{a}$ & $0,15 \pm 0,05 \mathrm{a}$ \\
\hline & \multirow[t]{3}{*}{$\mathbf{p H}$} & 5,8 & 24 & $20,85 \pm 11,00 \mathrm{a}$ & $0,22 \pm 0,11 \mathrm{a}$ & $0,20 \pm 0,10 \mathrm{a}$ \\
\hline & & 6,8 & 13 & $15,68 \pm 3,05 \mathrm{a}$ & $0,22 \pm 0,05 \mathrm{a}$ & $0,15 \pm 0,03 \mathrm{a}$ \\
\hline & & BAF & 7 & $21,16 \pm 4,39 a$ & $0,33 \pm 0,14 \mathrm{a}$ & $0,20 \pm 0,04 \mathrm{a}$ \\
\hline & \multirow{3}{*}{$\begin{array}{l}\text { Medio de } \\
\text { cultivo }\end{array}$} & EMA & 11 & $15,68 \pm 2,55 \mathrm{a}$ & $0,19 \pm 0,04 \mathrm{~b}$ & $0,15 \pm 0,02 \mathrm{a}$ \\
\hline & & $\mathrm{MMN}$ & 8 & $20,36 \pm 12,49 a$ & $0,16 \pm 0,09 b$ & $0,19 \pm 0,12 \mathrm{a}$ \\
\hline & & SAB & 15 & $18,95 \pm 11,37 \mathrm{a}$ & $0,22 \pm 0,07 \mathrm{~b}$ & $0,18 \pm 0,11 \mathrm{a}$ \\
\hline
\end{tabular}

Letras distintas en una misma columna para la misma variable indican diferencias significativas ( $\mathrm{p} \leq 0,05)$; DE: Desviación estándar. Letras diferentes na coluna, e para a mesma variável, indicam diferenças significativas $(p \leq 0,05)$; DE: O desvio padrão.

Al evaluar los resultados en función de la interacción de los factores, la situación se mantiene, encontrándose en la mayoría de los medios de cultivos, crecimientos más altos en los niveles de pH más ácidos, a excepción del medio EMA, medio con alta concentración de extracto de malta (rico en calorías, proteínas y glucósidos) y peptona, en que con un nivel de pH mayor (de 6,8), se alcanza también un mayor crecimiento, condición con la que se consigue además el crecimiento más alto del estudio con la cepa IF88003.

\subsubsection{Scleroderma citrinum}

Los niveles de $\mathrm{pH}$ y medios de cultivo no tuvieron un efecto importante en los crecimientos miceliares de S. citrinum ( $\mathrm{p}>0,05)$, sí se observan diferencias significativas en las cepas utilizadas $(p \leq 0,05)$, consiguiendo el mayor incremento con Sc8. No obstante lo anterior, entre los medios de cultivo el crecimiento medio radial más alto se alcanza en BAF (medio con mayor contenido de vitaminas y en menor medida cloruros y sulfatos) y MMN (medio con mayor concentración cloruros, y en menor concentración fosfatos, sulfatos, azucares y vitaminas) $\mathrm{y}$, entre los niveles de $\mathrm{pH}$, con un nivel intermedio se consiguen los mejores resultados (Tabla 2).

Cabe señalar que el crecimiento miceliar con esta especie fue bastante errático, observándose sólo en algunas pruebas y con un comportamiento mucho más lento que el mostrado por las cepas de S. luteus, situación que imposibilitó un análisis estadístico, sin embargo los valores que se obtienen con la cepa $\mathrm{Sc} 8$ son superiores a los obtenidos con la cepa IF84005, a pesar que esta última responde en todos los medios de cultivo y con los 3 niveles de $\mathrm{pH}$ (datos no publicados).

\subsection{Biomasa}

\subsubsection{Suillus luteus}

El análisis de datos indica que sólo en los medios de cultivo existe diferencia estadística entre los valores de biomasa, encontrándose la mejor respuesta en el medio BAF $(p \leq 0,05)$ (Tabla 2), y en general, si se incorpora el factor $\mathrm{pH}$, los mayores valores para esta variable se consiguen en los medios con presencia de vitaminas como la biotina e inositol, además de otros compuestos que mejoran las concentraciones de calcio, magnesio 
y fierro entre otros, y niveles más ácidos, a excepción del medio EMA que muestra una mejor respuesta con un nivel de $\mathrm{pH}$ más básico, estadísticamente diferenciable para las cepas IF81008 y IF83009 (Tabla 2).

\subsubsection{Scleroderma citrinum}

En esta especie sólo el medio de cultivo muestra un efecto importante en el volumen de biomasa que se produce, encontrándose la mejor respuesta con el medio BAF $(\mathrm{p} \leq 0,05)$ (Tabla 2).

\subsection{Velocidad de crecimiento}

\subsubsection{Suillus luteus}

Evaluando el comportamiento individual de cada cepa, la IF 88003 presentó la mayor velocidad de crecimiento. Por otro lado, con los niveles de pH más ácidos se observaron los mayores valores para esta variable, misma situación obtenida con los medios de cultivo BAF y EMA $(\mathrm{p} \leq 0,05)$ (Tabla 2$)$.

En los medios BAF y EMA la velocidad de crecimiento no se vio afectada mayormente por la diferencia de $\mathrm{pH}$, sin embargo el valor más alto se registró en el medio EMA con un $\mathrm{pH}$ de 6,8 , respuesta que se observó para cada una de las cepas $(\mathrm{p} \leq 0,05)$ (Tabla 3 ). Si bien no existen diferencias estadísticas entre los valores más altos mostrados por los medios MMNy $\mathrm{SAB}$ con respecto a los obtenidos en BAF y EMA, si se observa entre los niveles de $\mathrm{pH}$ en cada uno de ellos, lográndose las mayores velocidades con los niveles de $\mathrm{pH}$ más ácidos $(\mathrm{p} \leq 0,05)$.

\subsubsection{Scleroderma citrinum}

Se deriva de los registros de crecimiento radial que, entre las cepas evaluadas, la $\mathrm{Sc} 8$ presenta un valor de velocidad de crecimiento mayor $(\mathrm{p} \leq 0,05)$, no observándose diferencias entre los $\mathrm{pH}$ ni entre los distintos medios de cultivo evaluados (Tabla 2).

\section{DISCUSIÓN}

\subsection{Variables de comportamiento de Suillus luteus}

En esta especie, la cepa IF88003, de origen precordillerano, presentó un crecimiento radial superior al obtenido por las cepas de origen costero (IF81008) e intermedio (IF83009), lo que coincide con la evaluación realizada por Santelices et al. (2012), quienes observaron el crecimiento de tres cepas de S. luteus procedentes de la costa de la región del Maule, Chile, obteniendo los menores crecimientos en aquellas cepas más cercanas al borde costero, en las mismas condiciones que se mostraron propicias para cepas que crecen más hacia el interior.

En algunos casos las condiciones del medio pueden no provocar efectos importantes en el crecimiento de los hongos (SANTELICES et al., 2012), sin embargo, la incidencia de cambios de $\mathrm{pH}$ sobre una cepa se ha validado no solo para Suillus spp. (VÁSQUEZ et al., 2002), sino que también para cepas de Lactarius deliciosus L. ex Fr. Gray (FLORES et al., 2008). Pereira et al.(2007), por ejemplo, evaluaron el efecto del pH en medio de cultivo BAF en varios hongos, concluyendo que con $\mathrm{pH} 4,8$ y 5,8 se presentan los mayores valores de crecimiento radial para $S$. luteus, resultados que además concuerdan con lo obtenidoen este estudio. Con S. bellinii (Inzenga) O. Kuntze, se obtienen resultados similares logrando mayor velocidad y área de crecimiento con niveles de $\mathrm{pH}$ de 4,8 y 5,8 (SIANARD et al., 2010).

Asimismo, el tipo de medio de cultivo es otra condición que en general afecta el comportamiento de los hongos, aunque en algunas especies puede no provocar mayores cambios, situación observada por Díaz et al. (2009) para la especie Suillus tomentosus (Kauff.) Sing en distintos medios de cultivo. No obstante, de los medios de cultivo evaluados, con EMA y BAF se alcanzan valores superiores de crecimiento radial con respecto a los medios MMN y SAB, equivalente a lo observado en otros estudios con esta misma especie (FERNÁNDEZ-MIRANDA et al., 2008), como también en las especies Laccaria bicolor (Maire) P.D. Orton. (SANTIAGO-MARTÍNEZ et al., 2003) y Lactarius deliciosus (CHÁVEZ et al., 2008), para los mismos medios de cultivo.

En términos de biomasa, el medio de cultivo BAF presenta las mejores condiciones, situación que también se ha registrado para Laccaria bicolor (SANTIAGO-MARTÍNEZ et al., 2003). Respecto al efecto del pH, si bien los niveles de 4,8 y 5,8 presentaron los valores medios más altos estos no fueron estadísticamente distintos que los alcanzados con un nivel de 6,8. En este sentido, Vásquez et al. (2002) señalan en su investigación que, a un pH cercano a 6, cepas de Suillus spp. alcanzan la mayor producción de biomasa.

Revista Árvore, Viçosa-MG, v.39, n.1, p.105-113, 2015

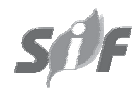


Tabla 3 - Valores medios \pm desviación estándar de crecimiento radial, velocidad de crecimiento y biomasa, de tres cepas de Suillus luteus procedentes de plantaciones forestales, desarrolladas en cuatro medios de cultivo y tres niveles de $\mathrm{pH}(\mathrm{n}=5)$.

Tabela 3 - Valores médios \pm desvio regular de crescimento radial, taxa de crescimento e biomassa de três estirpes de Suillus luteus de plantações florestais em quatro meios de cultura e três níveis de $p H(n=5)$.

\begin{tabular}{|c|c|c|c|c|c|c|c|c|c|c|c|c|}
\hline \multirow[b]{3}{*}{$\begin{array}{l}\text { Cepas/ } \\
\text { Variables }\end{array}$} & \multicolumn{12}{|c|}{ Medios de cultivo } \\
\hline & \multicolumn{3}{|c|}{$\mathrm{BAF}$} & \multicolumn{3}{|c|}{ EMA } & \multicolumn{3}{|c|}{$\mathrm{MMN}$} & \multicolumn{3}{|c|}{$\mathrm{SAB}$} \\
\hline & $\mathrm{pH} 4,8$ & pH 5,8 & pH 6,8 & $\mathrm{pH} 4,8$ & $\mathrm{pH} \mathrm{5,8}$ & pH 6,8 & pH 4,8 & $\mathrm{pH} \mathrm{5,8}$ & pH 6,8 & pH 4,8 & $\mathrm{pH} 5,8$ & $\mathrm{pH} 6,8$ \\
\hline \multicolumn{13}{|l|}{ IF81008 } \\
\hline $\begin{array}{l}\text { Crecimiento } \\
\text { radial }(\mathrm{mm})\end{array}$ & $\begin{array}{c}25,68 \pm \\
1,75 \mathrm{a}\end{array}$ & $\begin{array}{c}25,33 \pm \\
1,92 \mathrm{a}\end{array}$ & $\begin{array}{c}20,40 \pm \\
6,59 \mathrm{a}\end{array}$ & $\begin{array}{c}22,98 \pm \\
7,20 \mathrm{a}\end{array}$ & $\begin{array}{c}21,53 \pm \\
2,73 \mathrm{a}\end{array}$ & $\begin{array}{c}25,88 \pm \\
7,20 \mathrm{a}\end{array}$ & $\begin{array}{c}20,13 \pm \\
2,28 \mathrm{a}\end{array}$ & $\begin{array}{c}20,08 \pm \\
4,59 \mathrm{a}\end{array}$ & $\begin{array}{c}17,47 \pm \\
5,04 \mathrm{~b}\end{array}$ & $\begin{array}{c}16,94 \pm \\
0,86 \mathrm{~b}\end{array}$ & $\begin{array}{c}23,20 \pm \\
4,25 \mathrm{a}\end{array}$ & $\begin{array}{c}12,20 \pm \\
3,63 \mathrm{~b}\end{array}$ \\
\hline $\begin{array}{l}\text { Velocidad } \\
\text { crecimiento } \\
\text { (mm/día) }\end{array}$ & $\begin{array}{l}0,68 \pm \\
0,05 \mathrm{a}\end{array}$ & $\begin{array}{l}0,67 \pm \\
0,05 \mathrm{a}\end{array}$ & $\begin{array}{l}0,54 \pm \\
0,17 \mathrm{a}\end{array}$ & $\begin{array}{l}0,60 \pm \\
0,19 \mathrm{a}\end{array}$ & $\begin{array}{l}0,57 \pm \\
0,07 \mathrm{a}\end{array}$ & $\begin{array}{l}0,68 \pm \\
0,19 \mathrm{a}\end{array}$ & $\begin{array}{l}0,53 \pm \\
0,06 \mathrm{a}\end{array}$ & $\begin{array}{l}0,53 \pm \\
0,12 \mathrm{a}\end{array}$ & $\begin{array}{l}0,46 \pm \\
0,13 \mathrm{~b}\end{array}$ & $\begin{array}{l}0,45 \pm \\
0,02 \mathrm{~b}\end{array}$ & $\begin{array}{l}0,61 \pm \\
0,11 \mathrm{a}\end{array}$ & $\begin{array}{l}0,32 \pm \\
0,10 \mathrm{~b}\end{array}$ \\
\hline Biomasa (g) & $\begin{array}{l}0,16 \pm \\
0,02 \mathrm{a}\end{array}$ & $\begin{array}{l}0,18 \pm \\
0,02 \mathrm{a}\end{array}$ & $\begin{array}{l}0,12 \pm \\
0,05 \mathrm{~b}\end{array}$ & $\begin{array}{l}0,10 \pm \\
0,05 \mathrm{c}\end{array}$ & $\begin{array}{l}0,09 \pm \\
0,02 \mathrm{c}\end{array}$ & $\begin{array}{l}0,14 \pm \\
0,05 \mathrm{~b}\end{array}$ & $\begin{array}{l}0,07 \pm \\
0,01 \mathrm{c}\end{array}$ & $\begin{array}{l}0,07 \pm \\
0,03 \mathrm{c}\end{array}$ & $\begin{array}{l}0,05 \pm \\
0,02 \mathrm{c}\end{array}$ & $\begin{array}{l}0,06 \pm \\
0,03 \mathrm{c}\end{array}$ & $\begin{array}{l}0,11 \pm \\
0,02 \mathrm{~b}\end{array}$ & $\begin{array}{l}0,05 \pm \\
0,03 \mathrm{c}\end{array}$ \\
\hline \multicolumn{13}{|l|}{ IF83009 } \\
\hline $\begin{array}{l}\text { Crecimiento } \\
\text { radial }(\mathrm{mm})\end{array}$ & $\begin{array}{c}23,53 \pm \\
2,13 \mathrm{a}\end{array}$ & $\begin{array}{c}22,73 \pm \\
1,11 \mathrm{a}\end{array}$ & $\begin{array}{c}22,50 \pm \\
2,21 \mathrm{a}\end{array}$ & $\begin{array}{c}23,33 \pm \\
5,90 \mathrm{a}\end{array}$ & $\begin{array}{c}21,30 \pm \\
7,62 \mathrm{a}\end{array}$ & $\begin{array}{c}23,85 \pm \\
3,15 \mathrm{a}\end{array}$ & $\begin{array}{c}23,25 \pm \\
2,36 \mathrm{a}\end{array}$ & $\begin{array}{c}21,33 \pm \\
2,38 \mathrm{a}\end{array}$ & $\begin{array}{c}18,40 \pm \\
4,48 b\end{array}$ & $\begin{array}{c}25,53 \pm \\
0,86 \mathrm{a}\end{array}$ & $\begin{array}{c}24,88 \pm \\
0,64 \mathrm{a}\end{array}$ & $\begin{array}{c}14,48 \pm \\
0,69 b\end{array}$ \\
\hline $\begin{array}{l}\text { Velocidad } \\
\text { crecimiento } \\
(\mathrm{mm} / \text { día })\end{array}$ & $\begin{array}{l}0,62 \pm \\
0,06 \mathrm{a}\end{array}$ & $\begin{array}{l}0,60 \pm \\
0,03 \mathrm{a}\end{array}$ & $\begin{array}{l}0,59 \pm \\
0,06 \mathrm{a}\end{array}$ & $\begin{array}{l}0,61 \pm \\
0,16 \mathrm{a}\end{array}$ & $\begin{array}{c}0,56 \pm \\
0,2 \mathrm{a}\end{array}$ & $\begin{array}{l}0,63 \pm \\
0,08 \mathrm{a}\end{array}$ & $\begin{array}{l}0,61 \pm \\
0,06 \mathrm{a}\end{array}$ & $\begin{array}{l}0,56 \pm \\
0,06 \mathrm{a}\end{array}$ & $\begin{array}{l}0,48 \pm \\
0,12 \mathrm{~b}\end{array}$ & $\begin{array}{l}0,67 \pm \\
0,02 \mathrm{a}\end{array}$ & $\begin{array}{l}0,65 \pm \\
0,02 \mathrm{a}\end{array}$ & $\begin{array}{l}0,38 \pm \\
0,02 \mathrm{~b}\end{array}$ \\
\hline Biomasa (g) & $\begin{array}{l}0,16 \pm \\
0,06 \mathrm{a}\end{array}$ & $\begin{array}{l}0,15 \pm \\
0,03 \mathrm{a}\end{array}$ & $\begin{array}{l}0,13 \pm \\
0,05 \mathrm{a}\end{array}$ & $\begin{array}{c}0,1 \pm \\
0,05 \mathrm{~b}\end{array}$ & $\begin{array}{l}0,09 \pm \\
0,05 \mathrm{~b}\end{array}$ & $\begin{array}{l}0,11 \pm \\
0,03 \mathrm{a}\end{array}$ & $\begin{array}{c}0,07 \pm \\
0,003 \mathrm{~b}\end{array}$ & $\begin{array}{l}0,08 \pm \\
0,07 \mathrm{~b}\end{array}$ & $\begin{array}{l}0,07 \pm \\
0,03 \mathrm{~b}\end{array}$ & $\begin{array}{l}0,12 \pm \\
0,01 \mathrm{a}\end{array}$ & $\begin{array}{l}0,12 \pm \\
0,01 \mathrm{a}\end{array}$ & $\begin{array}{c}0,06 \pm \\
0,003 \mathrm{~b}\end{array}$ \\
\hline \multicolumn{13}{|l|}{ IF88003 } \\
\hline $\begin{array}{l}\text { Crecimiento } \\
\text { radial }(\mathrm{mm})\end{array}$ & $\begin{array}{c}24,48 \pm \\
2,14 \mathrm{~b}\end{array}$ & $\begin{array}{c}23,28 \pm \\
1,97 \mathrm{~b}\end{array}$ & $\begin{array}{c}23,68 \pm \\
2,10 \mathrm{~b}\end{array}$ & $\begin{array}{c}29,40 \pm \\
3,00 \mathrm{a}\end{array}$ & $\begin{array}{c}29,65 \pm \\
3,99 \mathrm{a}\end{array}$ & $\begin{array}{c}32,90 \pm \\
4,15 \mathrm{a}\end{array}$ & $\begin{array}{c}30,43 \pm \\
6,09 \mathrm{a}\end{array}$ & $\begin{array}{c}30,08 \pm \\
7,89 \mathrm{a}\end{array}$ & $\begin{array}{c}20,78 \pm \\
8,03 \mathrm{~b}\end{array}$ & $\begin{array}{c}28,84 \pm \\
1,18 \mathrm{a}\end{array}$ & $\begin{array}{c}26,35 \pm \\
4,65 \mathrm{a}\end{array}$ & $\begin{array}{c}14,70 \pm \\
2,60 \mathrm{c}\end{array}$ \\
\hline $\begin{array}{l}\text { Velocidad } \\
\text { crecimiento } \\
(\mathrm{mm} / \text { día })\end{array}$ & $\begin{array}{l}0,64 \pm \\
0,06 \mathrm{~b}\end{array}$ & $\begin{array}{l}0,61 \pm \\
0,05 \mathrm{~b}\end{array}$ & $\begin{array}{l}0,62 \pm \\
0,06 \mathrm{~b}\end{array}$ & $\begin{array}{l}0,77 \pm \\
0,08 \mathrm{a}\end{array}$ & $\begin{array}{l}0,78 \pm \\
0,11 \mathrm{a}\end{array}$ & $\begin{array}{l}0,87 \pm \\
0,11 \mathrm{a}\end{array}$ & $\begin{array}{l}0,80 \pm \\
0,16 \mathrm{a}\end{array}$ & $\begin{array}{l}0,79 \pm \\
0,21 \mathrm{a}\end{array}$ & $\begin{array}{l}0,55 \pm \\
0,21 \mathrm{~b}\end{array}$ & $\begin{array}{l}0,76 \pm \\
0,03 \mathrm{a}\end{array}$ & $\begin{array}{l}0,69 \pm \\
0,12 \mathrm{a}\end{array}$ & $\begin{array}{l}0,39 \pm \\
0,07 \mathrm{c}\end{array}$ \\
\hline Biomasa (g) & $\begin{array}{l}0,13 \pm \\
0,01 \mathrm{a}\end{array}$ & $\begin{array}{l}0,14 \pm \\
0,03 \mathrm{a}\end{array}$ & $\begin{array}{l}0,13 \pm \\
0,02 \mathrm{a}\end{array}$ & $\begin{array}{l}0,11 \pm \\
0,03 \mathrm{a}\end{array}$ & $\begin{array}{l}0,12 \pm \\
0,02 \mathrm{a}\end{array}$ & $\begin{array}{l}0,14 \pm \\
0,04 \mathrm{a}\end{array}$ & $\begin{array}{l}0,07 \pm \\
0,01 \mathrm{~b}\end{array}$ & $\begin{array}{l}0,07 \pm \\
0,02 \mathrm{~b}\end{array}$ & $\begin{array}{l}0,06 \pm \\
0,03 \mathrm{~b}\end{array}$ & $\begin{array}{l}0,14 \pm \\
0,05 \mathrm{a}\end{array}$ & $\begin{array}{l}0,11 \pm \\
0,02 \mathrm{a}\end{array}$ & $\begin{array}{l}0,05 \pm \\
0,02 \mathrm{~b}\end{array}$ \\
\hline
\end{tabular}

Letras distintas en una misma fila indican diferencias significativas entre medios de cultivo para cada cepa fúngica a un nivel $\mathrm{P}<0,05$. Letras diferentes na mesma linha indicam diferenças significativas entre os meios de cultura para cada cepa fúngica em $P<0,05$.

Como se señaló, la velocidad de crecimiento se estima en función del crecimiento radial para un período determinado, parámetro que en este estudio alcanza valores similares a los reportados por Pereira et al. (2007) con cepas de esta misma especie, pero inferiores a lo obtenido por Santelices et al. (2012) en su estudio con cepas de $S$. luteus de la región del Maule.

Las mayores velocidades de crecimiento conseguidos en los medios de cultivo EMA y BAF, son coincidentes con lo obtenido por Santiago-Martínez et al. (2003) con Laccaria bicolor para los mismos medio de cultivo. Por otro lado, los valores alcanzados según el nivel de $\mathrm{pH}$, que en general son mayores con niveles de pH más ácidos, concuerda con lo observado por Santelices et al. (2012).

\subsection{Variables de comportamiento Scleroderma citrinum}

Las cepas de esta especie presentaron un comportamiento muy distinto entre sí. Mientras la cepa más septentrional (IF84005) mostró crecimientos radiales en los cuatro medios de cultivo, con la mejor respuesta en los medios BAF y EMA, la cepa de más al sur (Sc8) sólo respondió a la reproducción en los medios $\mathrm{MMN}$ 
y SAB alcanzando a pesar de ello los valores más altos registrados entre ambas. En este sentido, FernándezMiranda et al. (2008) reportan resultados similares que lo obtenido con la cepa IF84005 para una cepa de la misma especie en un medio de cultivo BAF. Por otro lado, Malvárez et al. (1997) entregaron antecedentes de cepas de Scleroderma spp., asociados a Eucalyptus grandis Hill ex Maiden, con crecimientos radiales en medios de cultivo SAB y MMN, no habiendo diferencias significativas entre los incrementos obtenidos en ambos medios al cabo de 8 semanas.

Se señaló que, debido a lo errático de los resultados, no fue posible un análisis estadístico apropiado, no obstante, por los valores medios se observa para ambas cepas una mejor respuesta en crecimiento con un $\mathrm{pH}$ de 5,8 , inclusive con la cepa $\mathrm{Sc} 8$ sólo se obtiene crecimiento con este nivel de acidez para el medio de cultivo MMN. Vásquez et al. (2002) señalan en su estudio que, en la mayoría de las interacciones aplicadas a los medios para reproducción controlada de Scleroderma polyrhizum (J.F.Gmel.) Pers., el mayor valor de crecimiento miceliar se presenta con $\mathrm{pH}$ entre 5 y 6 . Sianard et al. (2010) evaluaron la influencia del pH en el desarrollo de cinco hongos ectomicorrícicos, entre ellos dos especies del género Scleroderma, comprobando para estas últimas que los mayores crecimientos se obtenían con medios de cultivos con $\mathrm{pH}$ de 5,8 y 6,8 .

Aunque sólo la cepa IF 84005 respondió a los 4 medios de cultivo, $\mathrm{Sc} 8$ sólo mostró crecimiento en $\mathrm{MMN}$ y SAB, aparece un efecto provocado por esta condición presentándose una mayor incidencia utilizando medio BAF por sobre los otros tres medios, más bien por el alto valor obtenido por esta cepa en ese medio de cultivo. Santiago-Martínez et al. (2003), logran para cepas de Laccaria bicolor los mayores valores de biomasa utilizando medio BAF, pero también altos valores en el medio de cultivo SAB, como ocurre también en el presente estudio con ambas cepas.

Por su parte, el efecto del pH, analizada como variable aislada, no obtuvo diferencia significativa entre niveles. El comportamiento se presentó con una mayor variedad, identificándose por ejemplo para la cepa IF84005 una mejor respuesta con un pH intermedio en los medios BAF y EMA, al contrario que en el medio MMN que con un pH más ácido se obtiene una mejor respuesta, y en el medio $\mathrm{SAB}$ con un $\mathrm{pH}$ más básico. Con la cepa $\mathrm{Sc} 8$, con un $\mathrm{pH}$ intermedio y básico se presentaron más apropiados para alcanzar mayores valores de biomasa. Vásquez et al. (2002), consiguen mayores valores de biomasa para cepas de Scleroderma polyrhizum con un $\mathrm{pH}$ entre 5 y 6 , al igual que lo alcanzado por Dennis (1985) en cepas de Scleroderma bovista Fries.

En relación con la velocidad de crecimiento, los resultados obtenidos en este estudios son similares a los señalados por Sánchez et al. (2000) para la especie Scleroderma verrucosum (Bull.) Pers., pero muy inferiores a los reportados por Sianard et al. (2010), quienes registraron velocidades de crecimiento para Scleroderma cepa Pers. de 1,92 mm/día, a un pH de 4,6, velocidades que disminuyeron a medida que aumentaba el nivel de $\mathrm{pH}$. De cualquier manera, los valores medios de velocidad alcanzados fueron más altos en todos los medios, y para las dos cepas, con $\mathrm{pH}$ de 5,8 .

\section{CONCLUSIÓN}

La masificación in vitro de cepas micorrícicas, asociadas a las principales especies forestales que se plantan en Chile, es factible. Los resultados indican que tanto el medio de cultivo, el pH del medio, así como las cepas de cada especie estudiada, son determinantes en las respuestas de crecimiento de los hongos ectomicorrícicos in vitro evaluados.

Las cepas de Suillus luteus se desarrollaron adecuadamente en un medio de cultivo con abundancia de nutrientes (BAF y MMN) como en baja presencia de ellos (EMA), y con pH más bien ácido $(4,8$ y 5,8$)$, no obstante, la cepa IF 88003 es la que presentó los niveles más altos de biomasa, crecimiento radial y velocidad de crecimiento. Por otro lado, las cepas de Scleroderma citrinum presentaron, para los mismos ambientes, un desarrollo inferior y lento, no obstante, la cepa $\mathrm{Sc} 8$ se reprodujo de forma óptima y rápida bajo un medio de cultivo BAF y con un $\mathrm{pH}$ moderadamente ácido de 5,8, por tanto, pareciera ser un nivel óptimo de acidez para la reproducción de esta micorriza.

\section{RECONOCIMIENTO}

El presente estudio se desarrolló en el marco del Proyecto Selección de Inóculos Biológicos y Desarrollo de Tecnologías para su Aplicación en Viveros Forestales (06CN12PFT-68), financiado por INNOVA Chile - CORFO y ejecutado por el Instituto Forestal de Chile.

Revista Árvore, Viçosa-MG, v.39, n.1, p.105-113, 2015 


\section{REFERENCIAS}

BRUNDRETT, M.; BOUGHER, N.; GROVE, T.; MALAJCZUK, N. Working with mycorrhizas in forestry and agriculture. Canberra: ACIAR, 1996.

CHÁVEZ, D.; PEREIRA, G.; MACHUCA, A. Crecimiento in vitro de Lactarius deliciosus en medio de cultivo BAF y MNM. In: CONGRESO CHILENO DE CIENCIAS FORESTALES, 2008, 4., 2008, Talca. Congreso... Talca: Universidad de Talca, 2008.

DENNIS, J. J. Effect of $\mathbf{p H}$ and temperature on in vitro growth of ectomycorrhizal fungi. Report BC-X-273. Vancouver: Canadian Forestry Service, Pacific Forestry Centre, 1985.

DÍAZ, G.; FLORES, R.; HONRUBIA, M. Descripción de cultivos miceliares de Boletales neotropicales y europeos (Boletus grupo edulis, Boletellus y Suillus) y formación de primordios de $B$. edulis en cultivo puro. Revista Mexicana de Micología, v.30, n.1, p.1-7, 2009.

DUÑABEITIA, M. K.; HORMILLA, S.; GARCÍAPLAZAOLA, J. I.; TXARTERINA, K.; ARTECHE, U.; BECERRIL, J. M. Differential responses of three fungal species to environmental factors and their role in the mycorrhization of Pinus radiata D. Don. Mycorrhiza, v. 14, n.1, p.11-18, 2004.

FERNÁNDEZ-MIRANDA, E.; ALVARADO, P.; ALONSO-GRAÑA, M.; MAJADA, J.; CASARES, A. Influencia de las condiciones culturales en el crecimiento de diferentes hongos ectomicorrícicos. Cuadernos de la Sociedad Española de Ciencias Forestales, v.28, p.207-211, 2008.

FLORES, R.; HONRUBIA, M.; DÍAZ, G.

Caracterización de cepas de Lactarius sección Deliciosi de Guatemala y su comparación con cepas europeas de L. deliciosus. Revista Mexicana de Micología, v.26, n.1, p.51-55, 2008.

GIANINAZZI-PERSON, V.; AZCÓN-AGUILAR. Fisiología de las micorrizas vesículo-arbusculares. In: OLIVARES, J.;BAREA, J.M. (Ed.). Fijación y movilización biológica de nutrientes. Granada: CSIC. II, 1991. p. 175-201.
HONRUBIA, M.; TORRES, P.; DÍAZ, G.; CANO, A. Manual para micorrizar plantas en viveros forestales. Madrid: Ministerio de Agricultura, Pesca y Alimentación, 1992.

INFOSTAT. InfoStat versión 2011. Grupo InfoStat. Córdoba: Facultad de Ciencias Agrarias, Universidad Nacional de Córdoba, 2011.

MALVÁREZ, G.; MAJOR, G.; CURBELO, V.; FRIONI, L. Hongos ectomicorrícicos en Eucalyptus grandis. Agrociencia, v.1, n.1, p.38-43, 1997.

MARX, D. H. The influence of ectotrophic fungi on the resistance of pine roots to pathogenic infections. I. Antagonism of mycorrhizal fungi to root pathogenic fungi and soil bacteria.

Phytopathology, v.59, p.153-163, 1969.

MOSER, M. Die Gattung Phlegmacium. Die Pilze Mitteleupora's 4. J. Bad Heilbrunn, 1960.

PERA, J.; PARLADÉ, J. Inoculación controlada con hongos ectomicorrícicos en la producción de planta destinada a repoblaciones forestales: Estado actual en España. Investigación Agraria. Sistemas y Recursos Forestales, v.14, n.3, p.419-433, 2005.

PEREIRA, G.; HERRERA, J.; MACHUCA, A.; SÁNCHEZ, M. Efecto del pH sobre el crecimiento in vitro de hongos ectomicorrícicos recolectados de plantaciones de Pinus radiata. Bosque, v.28, n.3, p.215-219, 2007.

SÁNCHEZ, F.; HONRUBIA, M.; TORRES, P. Características culturales de algunos hongos ectomicorrícicos en cultivo puro. Revista Iberoamericana de Micología, v.17, n.1, p.127-134, 2000.

SANTELICES, R.; ESPINOZA, S.; BRUNEL, N.; PALFNER, G. Effect of the geographical origin, culture media, and $\mathrm{pH}$ on the growth dynamic of the edible ectomycohrrizal mushroom Suillus luteus. Ciencia e Investigación Agraria, v.39, n.2, p.369-376, 2012.

SANTIAGO-MARTÍNEZ, G.; ESTRADA-TORRES, A.; VARELA, L.; HERRERA, T. Crecimiento en siete medios nutritivos y síntesis in vitro de una cepa de Laccaria bicolor. Agrociencia, v.37, n.6, p.575-584, 2003. 
SCOTT, A. J.; KNOTT, M. A cluster analysis method for grouping means in the analysis of variance. Biometrics, v.30, p.507-512, 1974.

SIANARD, F. D.; PANGOU, S. V.; MOUNTANDA, A. Influencia del $\mathrm{pH}$ en el desarrollo in vitro de cinco especies de hongos ectomicorrícicos.

Centro Agrícola, v.37, n.1, p.23-28, 2010.

SCHMID, V. Entwicklung molekularer methoden fft ein schnelles und kostenges unddu monitoring der inokulation von forstpflanzen mit ektomykorrhizasymbionten. 2006. Dissertation (PhD) - University of Innsbruck, Innsbruck, 2006
SMITH, S. E.; SMITH, F. A.; JAKOBSEN, I. Functional diversity in arbuscular mycorrhizal (AM) symbiosis: the contribution of the mycorrhizal P uptake pathway is not correlated with mycorrhizal responses in growth or total $\mathrm{P}$ uptake. New Phytologist, v.162, p.511-524, 2004.

VÁSQUEZ-GARCÍA, A.; SANTIAGO-MARTÍNEZ, G.; ESTRADA-TORRES, A. Influencia del pH en el crecimiento de quince cepas de hongos ectomicorrizógenos. Anales del Instituto de Biología, Universidad Nacional Autónoma de México. Serie Botánica, v.73, n.1, p.1-15, 2002. 